\title{
PRGF as adjunct to DBB in maxillary sinus floor augmentation: histological results of a pilot split-mouth study
}

\author{
Leonidas Batas $^{1 *}$, Lazaros Tsalikis ${ }^{1}$ and Andreas Stavropoulos ${ }^{2,3,4}$
}

\begin{abstract}
Background: Various technologies of autologous blood concentrates are currently evaluated for their potential to enhance bone formation.

Aim: To report on the histological outcome of maxillary sinus floor augmentation (MSFA) with deproteinized bovine bone (DBB) in combination with chair-side prepared autologous platelet-rich growth factor (PRGF), in comparison to that with DBB alone.

Materials and methods: Six partially edentulous patients with $\leq 3-\mathrm{mm}$ residual bone height bilaterally in the posterior maxilla were subjected to MSFA with the lateral window technique, using DBB in combination with PRGF (PRGF System1 Vitoria, Spain) on one side or DBB alone on the contralateral side. Cylindrical biopsies from the augmented sinuses were collected during implant installation, ca. 6 months post-MSFA, and subjected to non-decalcified histological and histomorphometric evaluation.

Results: The collected biopsies varied in length (range 3.5-9.9 mm); consequently, the portion of the biopsy representing augmented tissues also varied (range 2.3-14.6 $\mathrm{mm}^{2}$ ). New bone formation with a trabecular appearance and numerous DBB particles in contact with the new bone or with loose connective tissue were observed. No differences in the relative volumes of bone formation were found in sinuses augmented with DBB + PRGF or DBB alone 6 months after MSFA (35.6 $\pm 8.26 \mathrm{~mm}$ and $37.8 \pm 3.15 \mathrm{~mm}$, respectively).

Conclusion and clinical implications: In conclusion, based on these preliminary results, PRGF as adjunct to DBB for MSFA, except from improved handling during the operation, does not appear to enhance nor interfere with bone formation inside the human sinus 6 months after MSFA, compared with the use of DBB alone.
\end{abstract}

Keywords: PRGF, Deproteinized bovine bone, Sinus elevation, Histology, Humans

\section{Introduction}

Maxillary sinus floor augmentation (MSFA) is a standard procedure to re-establish adequate bone volume and ridge height for implant installation in the posterior maxilla. MSFA was introduced to the profession by Boyne in 1980 [1-3] and is a highly predictable technique with high graft and implant survival rates $[4,5]$. Initially, sinus elevation techniques relied on grafting with $100 \%$ autogenous bone, harvested from either oral (ramus, chin) or extraoral (iliac crest) donor sites [6-8]. It was subsequently found that

\footnotetext{
* Correspondence: Ibatas76@gmail.com

'Department of Preventive Dentistry, Periodontology and Implant Biology, School of Dentistry, Aristotle University of Thessaloniki, Thessaloniki, Greece Full list of author information is available at the end of the article
}

autogenous bone grafting can be replaced, in whole or in part, by a variety of bone substitutes, like allografts, xenografts, alloplasts, [9-11], alone, or in combination with growth factors [12-16].

During recent years, several technologies of chair-side, autologous blood concentrates have been proposed as growth factor (GF) sources [17]. These autologous blood concentrates consist of a volume of plasma enriched with a large number of platelets (i.e., platelet-rich constructs) which, after activation, they release GFs (e.g., platelet-derived growth factor (PDGF), transforming growth factor beta (TGF-b), insulin-like growth factor (IGF), vascular endothelial growth factor (VEGF), basic fibroblast growth factor (bFGF), and hepatocyte growth factors (HGF), with the potential to 
enhance bone healing/regeneration [13, 17-19, 20]. Plasma-rich in growth factors (PRGF) [21] is such autologous blood-derived concentrate and has been described to enhance bone formation in bone defects, including MSFA [21-24].

Nevertheless, PRGF has a gel-like consistency and thus lacks space-provision capacities; hence, it seems reasonable that in MSFA, PRGF needs to be combined with a space providing material, e.g., a bone substitute, in order to achieve adequate volume of augmentation. Among the most commonly used bone substitutes in oral surgical procedures is deproteinized bovine bone (DBB) [25, 26], and successful (histological and clinical) outcomes have been reported for MSFA with DBB alone or in combination with autogenous bone, cells, and/or growth factors [27-29]. In one human histological case series with five patients [30], PRGF + DBB resulted in increased vascularization and increased bone formation 5 months after MSFA compared with only DBB implantation.

Thus, the aim of the present report was to add information on the existing, rather scarce, evidence about the histological outcome of MSFA with DBB in combination with chair-side prepared PRGF compared with that of MSFA with DBB alone.

\section{Material methods}

Six patients, requiring bilateral sinus augmentation procedures prior to placement of implants and no contraindications for this procedure (e.g., uncontrolled diabetes, long-term steroid usage, and blood disorders) were selected from those presenting at the Aristotle University of Thessaloniki School of Dentistry, Department of Periodontology. All patients were advised of alternative treatment plans and selected the plan requiring maxillary sinus elevation. Further, the patients were informed of the requirements for participation in the study, and all had the option of withdrawing from the study at any time. The nature of the study was explained to each patient, and each signed an informed consent form that was approved by the University ethical Committee on Activities Involving Human Subjects of the University of Thessaloniki, Dental School (14/02-02-2017). Due to the pilot character of this study, no sample size or power calculation was performed and only a few patients in need for a bilateral sinus augmentation were included. The intention was to assess any trends for differences between groups, and thus to decide on the possible relevance of a larger-scale study.

\section{Surgical procedure}

On the day of surgery, a flip of a coin determined the side receiving PRGF (PRGF System 1, Vitoria, Spain) in combination with DBB (Bio-Oss/Geistlich Biomaterials, Switzerland); the contralateral side received only DBB.
Briefly, MSFA was performed with a lateral window approach, where after osteotomy, the lateral bone window was lifted upwards together with the Schneiderian membrane very carefully separated from the bone, and the bone graft material was placed into the newly created space (Fig. 1). All 12 sinuses in this pilot study were grafted with cancellous DBB particles 0.25 to $1 \mathrm{~mm}$ in size.

\section{PRGF preparation}

Twenty milliliters of peripheral blood was collected by venipuncture directly into tubes (BTI blood collecting tubes, BTI Vitoria, Spain) containing 3.8\% (wt/vol) sodium citrate as anticoagulant. The blood was then centrifuged at $1400 \mathrm{rpm}$ for $8 \mathrm{~min}$ at room temperature (PRGF System1, Vitoria, Spain), and thus separated into its three basic components: red blood cells (at the bottom of the tube); PRGF (in the middle of the tube); and plasma poor in growth factors (at the upper part of the tube). The $0.5 \mathrm{ml}$ PRGF fraction located just above the red cell fraction was collected, with care being taken to avoid the overlying buffy coat, and deposited in an Eppendorf tube. Clotting and activation were initiated by adding $50 \mu \mathrm{l}$ calcium chloride solution $(10 \% w / v)$ to the Eppendorf tube. The activated PRGF was then mixed with $1 \mathrm{cc}$ of DBB in a glass dish, and after 5-8 min the material became viscous and was ready for use (Fig. 2).

\section{Infection and pain control}

All patients were prescribed systemic antibiotics (2 $\mathrm{g}$ amoxicillin with clavulanic acid) starting 1 day before surgery and for 6 days post-surgically. Dexamethasone $(8 \mathrm{mg})$ was prescribed and administered orally right before the surgery and for the following 3 days with a decreasing dose $(8,4$, and $2 \mathrm{mg}$ respectively). Analgesics were also prescribed (600mgr ibuprofen) for pain control. The patients were then asked to describe the post-operative discomfort as no pain discomfort, pain controlled with painkillers, or great pain.

\section{Histologic and histomorphometric analyses}

Cylindrical biopsies were retrieved at the time of implant placement by means of trephine burs with an external/internal diameter of $3 \mathrm{~mm} / 2 \mathrm{~mm}$, about 6 months after MSFA (Fig. 3). Immediately after harvesting, the trephines containing the tissue cores were placed in $70 \%$ alcohol for fixation. The cores were code-masked to facilitate blind histological assessment. After 2 weeks, the biopsy core was removed from the trephine, whenever possible; otherwise, the entire trephine-biopsy complex was further processed for non-decalcified sectioning, including dehydration in a series of increasing concentrations of ethanol and embedding in methylmethacrylate. Longitudinal sections were generated with a cutting-grinding technique and then stained with van Gieson's picro-fuchsin. Histological and histomorphometrical analysis was performed while viewing 

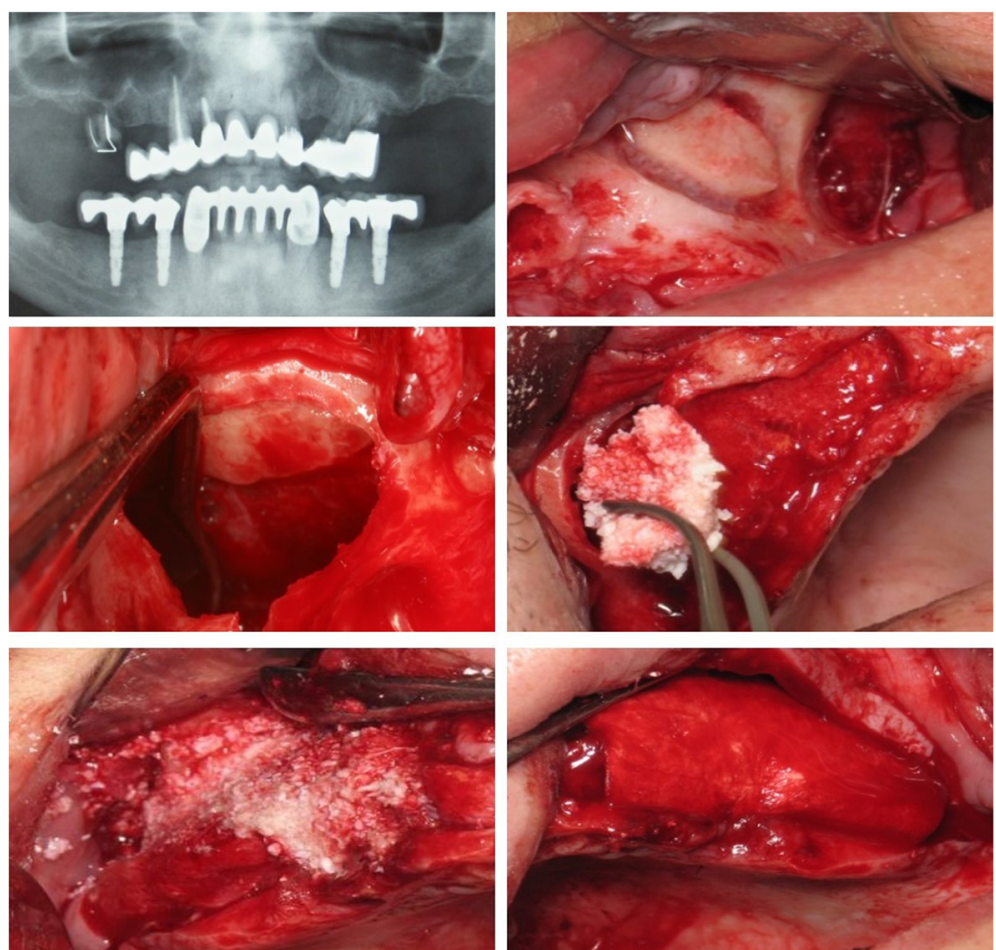

Fig. 1 Panoramic radiograph (a) of one patient receiving bi-lateral MSFA with the lateral window approach (b), where after sinus membrane elevation (c), one site was grafted with DBB + PRGF (d) and the other with DBB alone (e); the window was always covered with a collagen membrane

the most central section of each biopsy under a microscope with incandescent light (BH-50, Olympus, Ballerup, Denmark) fitted to a video camera (Olympus DP70, Olympus). First, the margin between pristine bone and newly formed tissue inside the sinus was histologically identified, and then the area fractions (\%) of newly formed bone (mineralized tissue and bone marrow), soft connective tissue, residual biomaterial, empty spaces, and debris were estimated by a semi-automated technique based on color segmentation through the software (Adobe Photoshop; Adobe) (Fig. 4).
Summary statistics were used to describe the data, and the two-sided Wilcoxon test for paired observations was used to evaluate differences in the various tissue components between the two groups. The significance level was set at $P<0.05$. The SPSS 13.0 software (SPPS Inc., Chicago, IL, USA) was used for the statistical analysis.

\section{Results}

Handling and placement of DBB into the sinus were considerably improved when combined with PRGF (Fig. 2). Further, most patients referred to increased pain and swelling associated with sites grafted with only DBB. No major
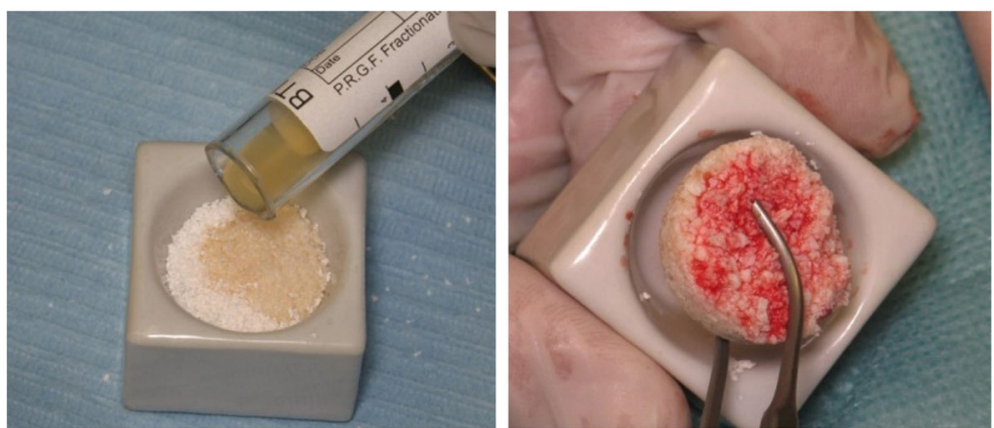

Fig. 2 PRGF is mixed with DBB (a) and after a few minutes the mixture attains a viscous consistency facilitating easy handling of the bone substitute particles (b) 

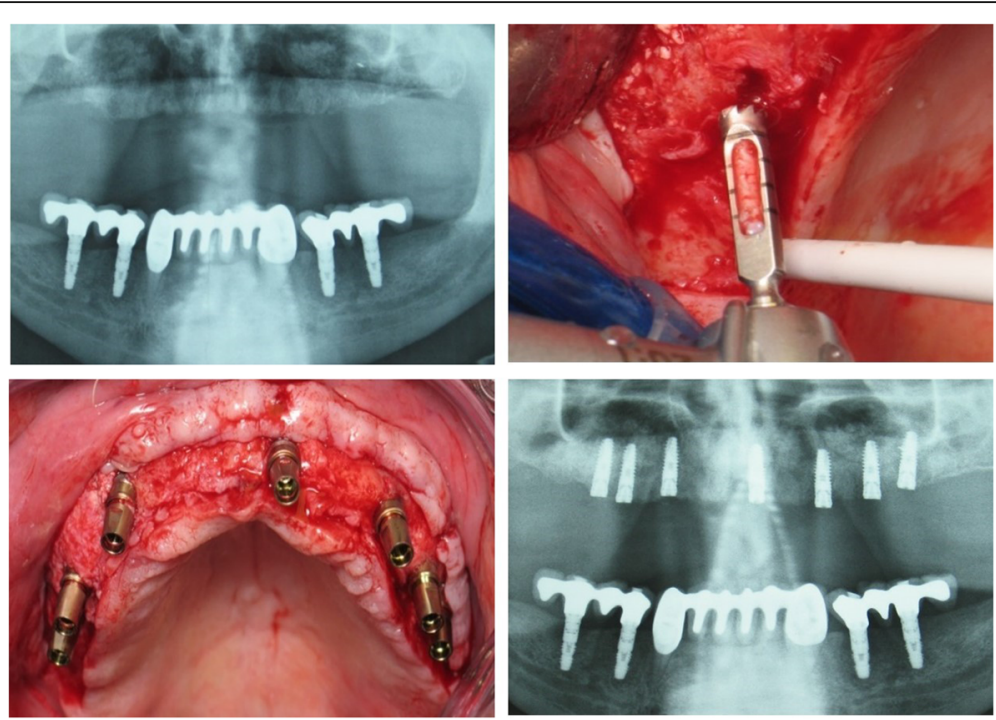

Fig. 3 Panoramic X-ray of the case presented in Fig. 2, 6 months post-operatively (a), showing increased radio-opacity in both grafted sinuses. Biopsies were obtained with trephine burs during oral implant site preparation (b)

adverse reactions were noted during MSFA or the post-operative phase, and no patients were excluded from the study. Further, all retrieved specimens could be processed and analyzed.

Variation in the length of the retrieved biopsy (range 3.5$9.9 \mathrm{~mm}$ ) was observed, and thus the portion of the biopsy representing augmented tissues also varied among biopsies (range $2.3-14.6 \mathrm{~mm}^{2}$ ); however, this was not obviously related to the original height of the residual ridge, neither with the amount of new bone formation within the biopsy. The biopsies consisted of a part including the cortical and trabecular bone of the residual alveolar ridge and a part corresponding to the new tissues formed in the sinus cavity. The new tissues consisted of variable amount of trabecular bone, DBB particles, loose connective tissue, and occasionally bone marrow. The newly formed bone was often in contact with the DBB particles, while no obvious signs of inflammation or foreign body reaction were observed.

There was no statistically significant difference between the two groups regarding any of the various tissue components within the sinus. The relative volume of new bone formation in sinuses augmented with DBB +

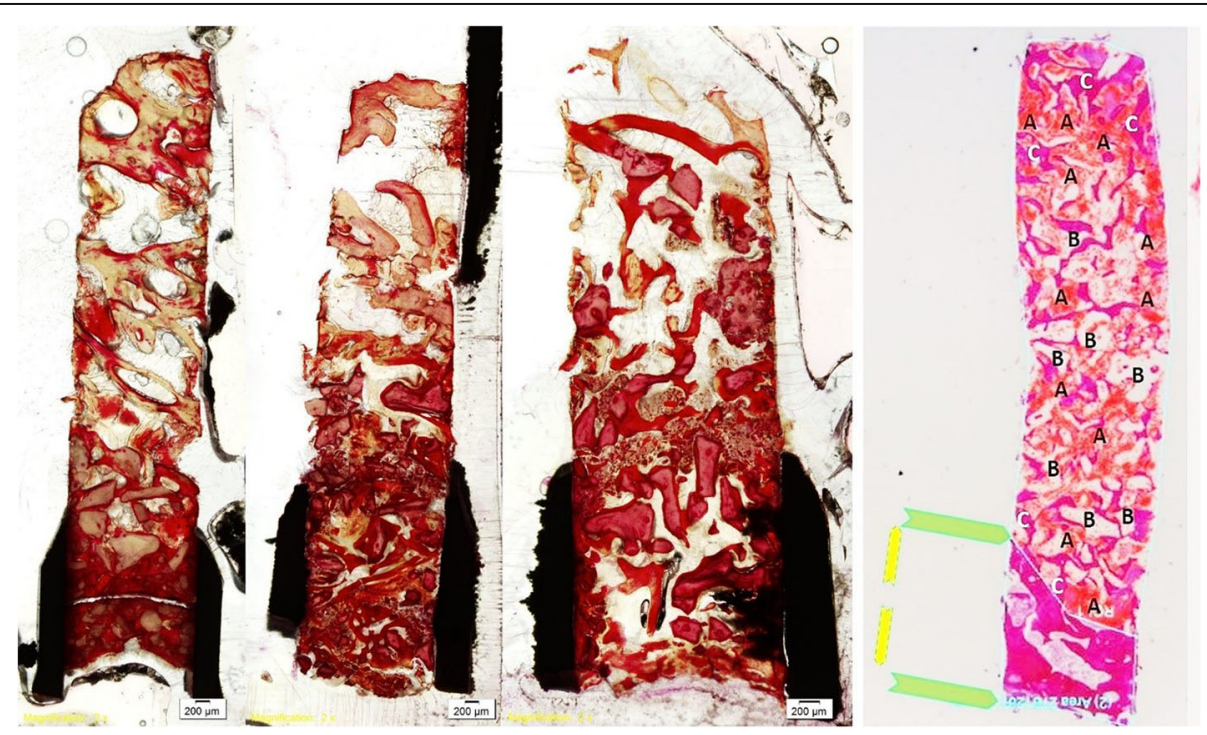

Fig. 4 The margin of pristine bone and augmented tissues was identified, and the various tissue components within the augmented area were semi-automatically estimated by dedicated image analysis software. Newly formed bone, connective tissue, DBB, within the augmented area, were identified and calculated 
Table 1 Relative volume of new bone formation

\begin{tabular}{|c|c|c|c|c|c|c|}
\hline \multirow[b]{2}{*}{$\begin{array}{l}N \\
\text { (patients) }\end{array}$} & \multicolumn{2}{|l|}{$\begin{array}{l}\text { New } \\
\text { bone (\%) }\end{array}$} & \multicolumn{2}{|l|}{$\begin{array}{l}\text { Connective } \\
\text { tissue (\%) }\end{array}$} & \multicolumn{2}{|l|}{$\begin{array}{l}\text { Particulates } \\
\text { DBB (\%) }\end{array}$} \\
\hline & $\mathrm{PRGF}+\mathrm{DBB}$ & DBB & $\mathrm{PRGF}+\mathrm{DBB}$ & DBB & $\overline{P R G F}+\mathrm{DBB}$ & DBB \\
\hline 1 & 43.86 & 40.95 & 24.02 & 26.52 & 32.12 & 32.53 \\
\hline 2 & 38.3 & 39.77 & 40.85 & 38.11 & 20.85 & 22.12 \\
\hline 3 & 35.47 & 37.9 & 34.26 & 36.80 & 30.26 & 23.95 \\
\hline 4 & 34.74 & 37.64 & 37.80 & 41.45 & 27.46 & 20.91 \\
\hline 5 & 33.87 & 35.89 & 33.55 & 28.95 & 32.58 & 35.16 \\
\hline 6 & 27.36 & 34.65 & 37.69 & 37.50 & 34.95 & 27.85 \\
\hline Mean & 35.6 & 37.8 & 34.69 & 34.88 & 29.7 & 27.08 \\
\hline SD & 8.26 & 3.15 & 5.86 & 5.81 & 5 & 5.79 \\
\hline
\end{tabular}

Histomorphometric characterization of the percentages of newly formed bone, connective tissue, and DBB particulates for each patient

PRGF was $35.6 \pm 8.26 \mathrm{~mm}$ and in sinuses augmented with DBB alone was $37.8 \pm 3.15 \mathrm{~mm}(P>0.05)$ (Table 1$)$.

\section{Discussion}

The results of this pilot study showed that PRGF as an adjunct to DBB grafting for MSFA did not enhance bone formation compared with DBB grafting alone, 6 months post-operatively. This result is directly in contrast with what presented in a report on a clinical study similar to the present one [30]. In this study, including five patients with MSFA, the same combination of PRGF + DBB resulted in increased vascularization and increased bone formation compared with only DBB implantation, 5 months post-op.

Indeed, conflicting results have been reported in both pre-clinical in vivo and clinical studies regarding the potential of PRGF to enhance bone regeneration. In several preclinical in vitro studies, bone defects treated with PRGF showed enhanced bone regeneration compared with controls. For example, PRGF implanted in extraction sockets, in humans, resulted in larger amounts of bone fill, compared with sockets left to heal alone [31], while narrow cylindrical defects in goat tibiae showed significantly larger amounts of mature bone trabeculae when treated with PRGF, than spontaneously healing sites [23]. In contrast, in other preclinical in vivo studies, adjunct use of PRGF did not promote bone regeneration or implant osseointegration, irrespective of the use or not of bone substitute materials, comparing to relevant controls [32-34]. Further, when PRGF was implanted in human extraction sockets, similar amounts of bone have been observed with those in spontaneously healed sockets $[35,36]$. Similarly conflicting results have been reported regarding the potential of other autologous blood preparations to enhance bone regeneration (e.g., PRP) [18, 37].

PRGF is suggested to be superior to other technologies of autologous blood concentrates, because of the unique preparation method, which results in (a) a high platelet concentration within the separated plasma (three times more than in peripheral blood) without white cell contamination, (b) slow release of GF over 7 days, when in other autologous blood concentrates systems (e.g., PRP) the release stops within $1 \mathrm{~h}$ when thrombin is used [17, 38, 39], (c) a leukocyte-free homogenous fibrin matrix, with reduced levels of proinflammatory cytokines interleukin IL-1 $\beta$ and IL-16. The present pilot experiment, however, was not designed to evaluate superiority of PRGF over other types of autologous blood preparations, and lack of any significant differences between the PRGF and control group in this pilot experiment do not necessarily imply lack of effect of the PRGF technology in MSFA procedures. Lack of additional effect of other types of growth factors in terms of bone regeneration when used as adjuncts to bone substitutes in MSFA has been observed in similar histological studies with similar or longer observation times as herein [11, 14, 40, 41]. For instance, in a study on bilateral MSFA, comparing DBB + PRP vs DBB alone, no benefit of the combined approach was observed [40], and in a recent systematic review of RCTs on sinus lift with or without adjunct use of PRP [42], the majority of studies failed to show a significant additive effect of PRP. In this context, the time point of post-op evaluation is critical in terms of interpreting the results of studies on biomaterials/adjuncts to enhance bone formation, i.e., extended observation times, may "wash-out" any possible positive effect of adjuncts on healing. Indeed, it appears that the major bulk of regenerated bone in sinuses grafted with bone substitutes forms within the first $4-5$ months, and thereafter only relatively small increase in bone formation may be observed [43, 44]. In the same line, in a recently published systematic review of histomorphometric data from human biopsies, sinuses grafted with autogenous bone and PRP showed larger average amounts of bone formation compared to sinuses grafted with autogenous bone alone, only at early observation times (i.e., $\leq 4.5$ months) and not at later time-points [45]. The differences between the abovementioned study [24] and the present one may thus be rather related to anatomical variation in sinus dimensions (e.g., width) [46] and/ to the method of histomorphometric analysis, rather than in the difference in the time-frame of healing (1 month).

In conclusion, based on these preliminary results, PRGF as adjunct to DBB for MSFA, except from improved handling during the operation, does not appear to enhance nor interfere with bone formation inside the human sinus 6 months after MSFA, compared with the use of DBB alone.

\section{Acknowledgements}

Not applicable.

Funding

The authors declare no funds for the research. 


\section{Availability of data and materials}

Mean data are available in Table 1. Pictures of histology cuts available upon request.

\section{Authors' contributions}

LB performed all surgeries on patients for the present study. LT analyzed the data collected. AS performed all histological measurements, and revised and approved the article. All authors read and approved the final manuscript.

\section{Ethics approval and consent to participate}

Patient recruitment and data collection of this study took place at the Aristotle University of Thessaloniki, School of Dentistry, Greece. The research was approved by the Ethics Committee of the Aristotle University of Thessaloniki, Greece (14/0202-2017) and all activities were conducted in full accordance with ethical principles, including the World Medical Association Declaration of Helsinki. We described the purpose of the study to patients, and the data were obtained anonymously.

\section{Consent for publication}

\section{Not applicable.}

\section{Competing interests}

Authors Batas Leonidas, Tsalikis Lazaros, and Stavropoulos Andreas declare that they have no competing interests.

\section{Publisher's Note}

Springer Nature remains neutral with regard to jurisdictional claims in published maps and institutional affiliations.

\section{Author details}

${ }^{1}$ Department of Preventive Dentistry, Periodontology and Implant Biology, School of Dentistry, Aristotle University of Thessaloniki, Thessaloniki, Greece. ${ }^{2}$ Department of Periodontology, Faculty of Odontology, Malmö University, Malmö, Sweden. ${ }^{3}$ Center for Experimental and Preclinical Biomedical Research (CEPBR), Athens, Greece. ${ }^{4}$ Division of Conservative Dentistry and Periodontology, University Clinic of Dentistry, Medical University of Vienna, Vienna, Austria.

\section{Received: 13 December 2018 Accepted: 5 February 2019}

\section{Published online: 01 April 2019}

\section{References}

1. Boyne PJ, James RA. Grafting of the maxillary sinus floor with autogenous marrow and bone. J Oral Surg. 1980;38(8):613-6.

2. Summers RB. The osteotome technique: Part 3--Less invasive methods of elevating the sinus floor. Compendium. 1994;15(6):698-700 702-4 passim; quiz 710

3. Toffler M. Site development in the posterior maxilla using osteocompression and apical alveolar displacement. Compend Contin Educ Dent. 2001;22(9):775-80 782, 784 passim; quiz 790.

4. Del Fabbro $M$, et al. Systematic review of survival rates for implants placed in the grafted maxillary sinus. Int J Periodontics Restorative Dent. 2004;24(6):565-77.

5. Shanbhag S, Shanbhag V, Stavropoulos A. Volume changes of maxillary sinus augmentations over time: a systematic review. Int J Oral Maxillofac Implants. 2014;29(4):881-92.

6. Wood RM, Moore DL. Grafting of the maxillary sinus with intraorally harvested autogenous bone prior to implant placement. Int J Oral Maxillofac Implants. 1988:3(3):209-14.

7. Raghoebar GM, et al. Augmentation of the maxillary sinus floor with autogenous bone for the placement of endosseous implants: a preliminary report. J Oral Maxillofac Surg. 1993;51(11):1198-203 discussion 1203-5.

8. Chanavaz M. Sinus grafting related to implantology. Statistical analysis of 15 years of surgical experience (1979-1994). J Oral Implantol. 1996;22(2):119-30.

9. Hallman M, Sennerby L, Lundgren S. A clinical and histologic evaluation of implant integration in the posterior maxilla after sinus floor augmentation with autogenous bone, bovine hydroxyapatite, or a 20:80 mixture. Int J Oral Maxillofac Implants. 2002;17(5):635-43.

10. Stavropoulos A, Karring T, Kostopoulos L. Fully vs. partially rough implants in maxillary sinus floor augmentation: a randomized-controlled clinical trial. Clin Oral Implants Res. 2007;18(1):95-102.
11. Stavropoulos A, et al. Histological evaluation of healing after transalveolar maxillary sinus augmentation with bioglass and autogenous bone. Clin Oral Implants Res. 2012;23(1):125-31.

12. Boyne PJ, et al. A feasibility study evaluating rhBMP-2/absorbable collagen sponge for maxillary sinus floor augmentation. Int J Periodontics Restorative Dent. 1997;17(1):11-25.

13. Marx RE, et al. Platelet-rich plasma: growth factor enhancement for bone grafts. Oral Surg Oral Med Oral Pathol Oral Radiol Endod. 1998;85(6):638-46.

14. Stavropoulos A, et al. Histological evaluation of maxillary sinus floor augmentation with recombinant human growth and differentiation factor5-coated beta-tricalcium phosphate: results of a multicenter randomized clinical trial. J Clin Periodontol. 2011;38(10):966-74.

15. Khairy NM, et al. Effect of platelet rich plasma on bone regeneration in maxillary sinus augmentation (randomized clinical trial). Int J Oral Maxillofac Surg. 2013:42(2):249-55.

16. Torrecillas-Martinez $L$, et al. Effect of rhBMP-2 upon maxillary sinus augmentation: a comprehensive review. Implant Dent. 2013:22(3):232-7.

17. Anitua $E$, et al. Autologous platelets as a source of proteins for healing and tissue regeneration. Thromb Haemost. 2004;91(1):4-15.

18. Marx RE. Platelet-rich plasma: evidence to support its use. J Oral Maxillofac Surg. 2004;62(4):489-96.

19. Lucarelli $E$, et al. Platelet-derived growth factors enhance proliferation of human stromal stem cells. Biomaterials. 2003:24(18):3095-100.

20. Leitner GC, et al. Platelet content and growth factor release in platelet-rich plasma: a comparison of four different systems. Vox Sang. 2006;91(2):135-9.

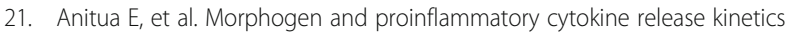
from PRGF-Endoret fibrin scaffolds: evaluation of the effect of leukocyte inclusion. J Biomed Mater Res A. 2015;103(3):1011-20.

22. Anitua $E$, et al. The potential impact of the preparation rich in growth factors (PRGF) in different medical fields. Biomaterials. 2007:28(31):4551-60.

23. Anitua $E$, et al. The effects of PRGF on bone regeneration and on titanium implant osseointegration in goats: a histologic and histomorphometric study. J Biomed Mater Res A. 2009;91(1):158-65.

24. Anitua $E$, Troya M, Orive $G$. Plasma rich in growth factors promote gingival tissue regeneration by stimulating fibroblast proliferation and migration and by blocking transforming growth factor-beta1-induced myodifferentiation. J Periodontol. 2014;83(8):1028-37.

25. Stavropoulos A, Karring T. Five-year results of quided tissue regeneration in combination with deproteinized bovine bone (bio-Oss) in the treatment of intrabony periodontal defects: a case series report. Clin Oral Investig. 2005; 9(4):271-7.

26. Stavropoulos A, Karring T. Guided tissue regeneration combined with a deproteinized bovine bone mineral (Bio-Oss) in the treatment of intrabony periodontal defects: 6-year results from a randomized-controlled clinical trial. J Clin Periodontol. 2010;37(2):200-10.

27. Jensen $\mathrm{T}$, et al. Maxillary sinus floor augmentation with Bio-Oss or Bio-Oss mixed with autogenous bone as graft in animals: a systematic review. Int J Oral Maxillofac Surg. 2012;41(1):114-20.

28. Jensen T, et al. Maxillary sinus floor augmentation with Bio-Oss or Bio-Oss mixed with autogenous bone as graft: a systematic review. Clin Oral Implants Res. 2012:23(3):263-73.

29. Hermund NU, et al. Reimplantation of cultivated human bone cells from the posterior maxilla for sinus floor augmentation. Histological results from a randomized controlled clinical trial. Clin Oral Implants Res. 2012;23(9):1031-7.

30. Anitua E, Prado R, Orive G. Bilateral sinus elevation evaluating plasma rich in growth factors technology: a report of five cases. Clin Implant Dent Relat Res. 2012;14(1):51-60.

31. Anitua E. Plasma rich in growth factors: preliminary results of use in the preparation of future sites for implants. Int J Oral Maxillofac Implants. 1999; 14(4):529-35.

32. Casati $M Z$, et al. Platelet-rich plasma does not improve bone regeneration around peri-implant bone defects - a pilot study in dogs. Int J Oral Maxillofac Surg. 2007:36(2):132-6.

33. Guerra I, et al. Evaluation of implant osseointegration with different regeneration techniques in the treatment of bone defects around implants: an experimental study in a rabbit model. Clin Oral Implants Res. 2011;22(3): 314-22.

34. Batas $L$, et al. Evaluation of autogenous PRGF+beta-TCP with or without a collagen membrane on bone formation and implant osseointegration in large size bone defects. A preclinical in vivo study. Clin Oral Implants Res. 2016;27(8):981-7. 
35. Gurbuzer B, et al. Scintigraphic evaluation of osteoblastic activity in extraction sockets treated with platelet-rich fibrin. J Oral Maxillofac Surg. 2010;68(5):980-9

36. Farina $\mathrm{R}$, et al. Plasma rich in growth factors in human extraction sockets: a radiographic and histomorphometric study on early bone deposition. Clin Oral Implants Res. 2013;24(12):1360-8.

37. Boyapati L, Wang HL. The role of platelet-rich plasma in sinus augmentation: a critical review. Implant Dent. 2006;15(2):160-70.

38. Miron RJ, et al. Use of platelet-rich fibrin in regenerative dentistry: a systematic review. Clin Oral Investig. 2017;21(6):1913-27.

39. Lemons PP, Chen D, Whiteheart SW. Molecular mechanisms of platelet exocytosis: requirements for alpha-granule release. Biochem Biophys Res Commun. 2000;267(3):875-80.

40. Froum SJ, et al. Effect of platelet-rich plasma on bone growth and osseointegration in human maxillary sinus grafts: three bilateral case reports. Int J Periodontics Restorative Dent. 2002;22(1):45-53.

41. Nery JC, et al. beta-TCP/HA with or without enamel matrix proteins for maxillary sinus floor augmentation: a histomorphometric analysis of human biopsies. Int J Implant Dent. 2017;3(1):18.

42. Pocaterra $A$, et al. Effectiveness of platelet-rich plasma as an adjunctive material to bone graft: a systematic review and meta-analysis of randomized controlled clinical trials. Int J Oral Maxillofac Surg. 2016;45(8): 1027-34.

43. Handschel J, et al. A histomorphometric meta-analysis of sinus elevation with various grafting materials. Head Face Med. 2009;5:12.

44. Klijn RJ, et al. A meta-analysis of histomorphometric results and graft healing time of various biomaterials compared to autologous bone used as sinus floor augmentation material in humans. Tissue Eng Part B Rev. 2010; 16(5):493-507.

45. Danesh-Sani SA, Engebretson SP, Janal MN. Histomorphometric results of different grafting materials and effect of healing time on bone maturation after sinus floor augmentation: a systematic review and meta-analysis. J Periodontal Res. 2017;52(3):301-12.

46. Bertl K, Domic D, Hirtler L, Heimel P, Esfandeyari A, Stavropoulos A, Ulm C. Micro- $C T$ evaluation of the cortical bone micro-architecture in the anterior and posterior maxilla and the maxillary sinus floor. Clin Oral Implants Res 2018;29(12):1220-9.

\section{Submit your manuscript to a SpringerOpen ${ }^{\circ}$ journal and benefit from:}

- Convenient online submission

- Rigorous peer review

- Open access: articles freely available online

- High visibility within the field

- Retaining the copyright to your article

Submit your next manuscript at $\boldsymbol{\nabla}$ springeropen.com 